\title{
Orbital effects on tunneling anisotropic magnetoresistance in Fe/GaAs/Au junctions
}

\author{
M. Wimmer, ${ }^{1}$ M. Lobenhofer, ${ }^{2}$ J. Moser, ${ }^{2}$ A. Matos-Abiague, ${ }^{1}$ D. Schuh, ${ }^{2}$ W. Wegscheider, ${ }^{2}$ J. Fabian, ${ }^{1}$ K. Richter, ${ }^{1}$ and \\ D. Weiss ${ }^{2}$ \\ ${ }^{1}$ Institut für Theoretische Physik, Universität Regensburg, 93040 Regensburg, Germany \\ ${ }^{2}$ Institut für Experimentelle und Angewandte Physik, Universität Regensburg, 93040 Regensburg, Germany
}

(Received 29 June 2009; published 1 September 2009)

\begin{abstract}
We report experiments on epitaxially grown Fe/GaAs/Au tunnel junctions demonstrating that the magnitude of the tunneling anisotropic magnetoresistance (TAMR) effect can be controlled by magnetic field strength. Theoretical modeling shows that the interplay of the orbital effects of a magnetic field and the Dresselhaus spin-orbit coupling in the GaAs barrier leads to an independent contribution to the TAMR effect with uniaxial symmetry, whereas the Bychkov-Rashba spin-orbit coupling does not play a role. The effect is intrinsic to barriers with bulk inversion asymmetry.
\end{abstract}

DOI: 10.1103/PhysRevB.80.121301

PACS number(s): 72.25.Dc, 75.47. $-\mathrm{m}$

Magnetic tunnel junctions (MTJs) are prominent examples of spintronic devices ${ }^{1,2}$ and have reached already technological importance. ${ }^{3}$ Typically, the resistance of a MTJ depends on the relative orientation of two ferromagnetic layers. ${ }^{1,2}$ Hence it came as a surprise when experiments on MTJs with only one ferromagnetic GaMnAs layer showed a sizable spin valve effect. ${ }^{4}$ Since then, this tunneling anisotropic magnetoresistance (TAMR) effect has been observed in tunnel junctions involving various materials ${ }^{5-9}$ as well as nanoconstrictions and break junctions. ${ }^{9-11}$ Amongst these experiments, the TAMR effect in Fe/GaAs/Au MTJs (Ref. 7) stands out due to its qualitatively different origin: whereas the TAMR effect usually originates from properties of the magnetic layer, namely, a spin-orbit induced anisotropic density of states ${ }^{4-6,8,10}$ in the ferromagnet or surface states, ${ }^{12-14}$ the TAMR in the Fe/GaAs/Au MTJ was attributed to an interference of Bychkov-Rashba spin-orbit coupling (SOC) at the barrier interface and the Dresselhaus SOC inside the barrier, i.e., to properties of the tunneling process itself. Moreover, the size and sign of the effect in this MTJ can be tuned by the bias voltage.

In this Rapid Communication, we show experimentally that the TAMR in Fe/GaAs/Au MTJs can also be controlled by a magnetic field. Our theoretical calculations ascribe this effect to an interplay of the orbital effects of the magnetic field and the Dresselhaus SOC in the GaAs barrier. This interplay leads to an independent TAMR contribution with uniaxial symmetry and is intrinsic to semiconductor barriers with bulk inversion asymmetry. In contrast to the usual tuning of spin-orbit effects through the electric field dependence of the Bychkov-Rashba SOC ${ }^{1,2}$ (bias voltage in the case of the $\mathrm{TAMR}^{7}$ ), here we demonstrate the control of a spin-orbit effect, namely the TAMR, through a magnetic field and the Dresselhaus SOC only; curiously the Bychkov-Rashba SOC does not play a role in this respect. As we show below, this is due to the different symmetries of the SOCs. Furthermore, in our analysis we find it important to include the orbital effects of the magnetic field in both the kinetic and SOC terms of the Hamiltonian, as both terms give rise to large competing contributions, resulting in a net TAMR effect in good agreement with experiment.

The type of tunneling device studied here is sketched in Fig. 1. We explored eight different samples all showing the same orbital effects discussed below. We hence focus here on one sample which consists of a 13-nm-thick Fe layer, grown epitaxially on a 8-nm-thick GaAs-tunneling barrier, and a $\mathrm{Au}$ top electrode. ${ }^{7}$ The GaAs barrier was grown by molecular beam epitaxy on sacrificial AlGaAs layers and capped with As to prevent oxidation during transport to a UHV magnetron sputtering system. There the As cap was removed at $T$ $=250{ }^{\circ} \mathrm{C}$ and $\mathrm{Fe}$ was grown at room temperature. Epitaxial growth of the Fe film was monitored by in situ reflection high-energy electron diffraction (RHEED). The Fe-layer is finally covered with $50 \mathrm{~nm} \mathrm{Co}$, and $150 \mathrm{~nm} \mathrm{Au}$ and serves as back contact. To prepare the top Au contact on the other side of the GaAs tunnel barrier, the wafer is glued upside down to another substrate and the original substrate is etched away. By employing optical lithography, selective etching and UHV-magnetron sputtering a circular, $13-\mu \mathrm{m}$-wide and 100nm-thick Au contact is fabricated.

The measurements were carried out at a temperature of $4.2 \mathrm{~K}$ inside a variable temperature insert of a ${ }^{4} \mathrm{He}$ cryostat. The device was placed in a rotatable sample holder allowing a $360^{\circ}$ in-plane rotation in the magnetic field $\mathbf{B}$ of a superconducting solenoid. The direction of $\mathbf{B}$ with respect to the hard axis of the Fe layer in [110] direction (nomenclature with respect to GaAs crystallographic directions) is given by the angle $\phi$ (Fig. 1). The resistance drop across the tunnel barrier was measured in four-point configuration using a HP $4155 \mathrm{~A}$ semiconductor parameter analyzer with the Aucontact grounded.

To measure the TAMR we rotated the sample by $180^{\circ}$ in a constant external magnetic field. The magnetic field strength was always high enough to align the magnetization
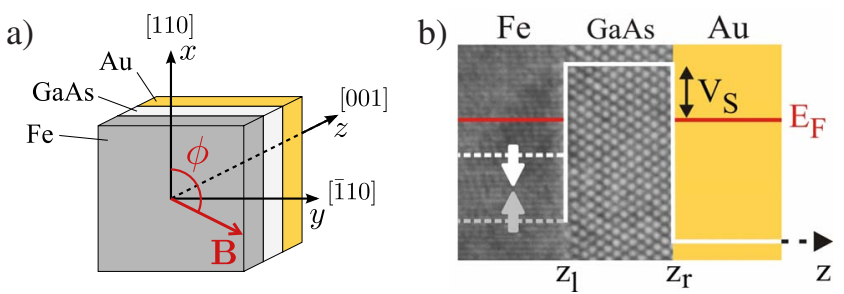

FIG. 1. (Color online) (a) Sketch of the Fe/GaAs/Au MTJ. (b) Schematic of the conduction-band profile. The gray background is a transmission electron micrograph of an epitaxial Fe/GaAs interface displaying the 8-nm-thick GaAs barrier. 

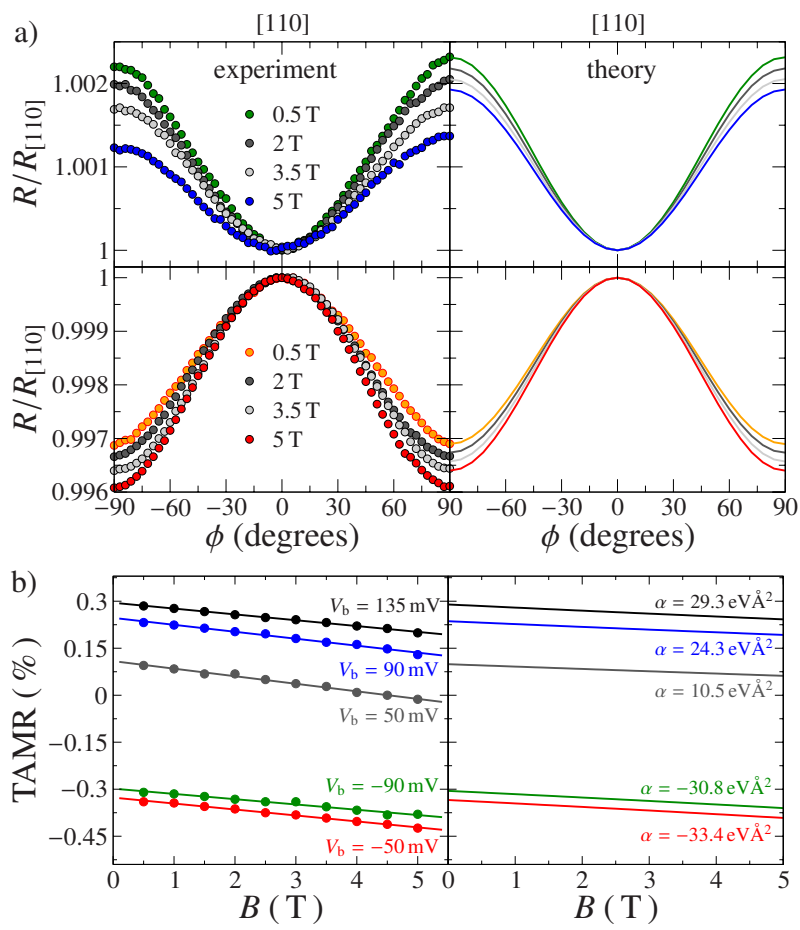

FIG. 2. (Color online) Comparison of experimental results (left panels) and numerical simulations (right panels) for the TAMR. (a) Angular dependence of $R(\phi)$ at various magnetic fields for an applied bias voltage of $V_{\mathrm{b}}=90 \mathrm{mV}$ (upper panels) and $V_{\mathrm{b}}=-90 \mathrm{mV}$ (lower panels). (b) $B$ dependence of the TAMR ratio [Eq. (1)] for different bias voltages. Lines in the left panel are a linear fit to the experimental data, shown as dots.

M along B. Figure 2(a) shows the results of such $\phi$ scans for various values of the magnetic field between 0.5 and $5 \mathrm{~T}$ and the two bias voltages, $+90 \mathrm{mV}$ (upper left panel) and -90 $\mathrm{mV}$ (lower left panel). The TAMR $R(\phi) / R_{[110]}$ shows the distinct uniaxial anisotropy characteristic for this system. ${ }^{7}$ As demonstrated recently, the TAMR strongly depends on the applied bias voltage and is connected to a bias dependent sign and strength of the Bychkov-Rashba parameter. ${ }^{7}$ For $\mathbf{M} \|[110]$ we always get a resistance maximum for $+90 \mathrm{mV}$ but a minimum for $-90 \mathrm{mV}$. This behavior is in accord with the one observed by Moser et al. ${ }^{7}$ and occurs for all samples investigated. In the simplest model the TAMR $R(\phi) / R_{[110]}-1 \sim \alpha \gamma[\cos (2 \phi)-1]$, where $\alpha$ and $\gamma$ are the Bychkov-Rashba and Dresselhaus parameters. While $\gamma$ is a material parameter, $\alpha$ is obtained by fitting the angular dependence $R(\phi) / R_{[110]}$ (see below).

With increasing magnetic field strength both the traces for positive and negative bias voltages are bent towards lower resistance values. If we define the TAMR ratio as

$$
\mathrm{TAMR}=\frac{R_{[110]}^{-}-R_{[110]}}{R_{[110]}},
$$

in which $R_{[110]}$ is the resistance for $\phi=+90^{\circ}$, the magnitude of the TAMR ratio decreases for positive bias voltages but increases for negative ones. This TAMR value measured as function of $B$ is displayed in the left panel of Fig. 2(b) for magnetic field strengths up to $5 \mathrm{~T}$. Note that the TAMR vanishes for a bias voltage of $+50 \mathrm{mV}$ at about $4.5 \mathrm{~T}$ but reappears again upon further increasing $B$. The magnetic field dependence of the TAMR ratio is in all cases linear. The slope $\Delta \mathrm{TAMR} / \Delta B$ of the best-fit line is nearly the same for all bias voltages indicating that the $B$ dependence of the TAMR is independent of the applied voltage. The experimental data in Fig. 2 are compared to model calculations discussed below.

The importance of orbital effects for charge tunneling has been pointed out already in the literature. ${ }^{15}$ Here, we focus on orbital effects on spin-dependent tunneling. In order to explain the experimental findings, we employ the spin-orbit based model for the TAMR effect of Refs. 2, 7, and 16 and include the orbital effects of the magnetic field. We choose the coordinate system such that the $x, y$, and $z$ directions are along the [110], [100], and [001] crystallographic directions and consider an in-plane magnetic field $\mathbf{B}=B \mathbf{n}$, where $\mathbf{n}=(\cos \phi, \sin \phi, 0)$ is a unit vector forming an angle $\phi$ with the $x$ axis [see Fig. 1(a)]. The Hamiltonian is given as $H=H_{0}+H_{\mathrm{BR}}+H_{\mathrm{D}}$, where

$$
H_{0}=+\frac{1}{2} \boldsymbol{\pi} \frac{1}{m^{*}(z)} \boldsymbol{\pi}+V(z)+\frac{\Delta(z)}{2} \mathbf{n} \cdot \boldsymbol{\sigma} .
$$

Here, $\boldsymbol{\pi}=-i \hbar \boldsymbol{\nabla}+e \mathbf{A}$, where $\mathbf{A}$ is the magnetic vector potential and $-e$ the electron charge. $m^{*}(z)$ is a position-dependent effective mass with $m^{*}(z)=0.067 m_{\mathrm{e}}$ in the GaAs barrier and $m^{*}(z)=m_{\mathrm{e}}$ in the Fe and Au layer, where $m_{\mathrm{e}}$ denotes the bare electron mass. $V(z)$ is the conduction-band profile in growth direction z. The GaAs Schottky barrier height is given by $V_{\mathrm{S}}=0.75 \mathrm{eV}$. The ferromagnetism in the Fe layer is described in terms of a Stoner model ${ }^{17}$ with spin splitting $\Delta(z)$. $\Delta(z)$ and $V(z)$ are chosen such that the Fermi wave vector in $\mathrm{Fe}$ is $k_{\mathrm{F}, \mathrm{Fe}}^{\uparrow}=1.05 \times 10^{-10} \mathrm{~m}^{-1}$ and $k_{\mathrm{F}, \mathrm{Fe}}^{\downarrow}=0.44 \times 10^{-10} \mathrm{~m}^{-1}$ for majority and minority electrons, ${ }^{18}$ respectively, and in $\mathrm{Au}$ $k_{\mathrm{F}, \mathrm{Au}}=1.2 \times 10^{-10} \mathrm{~m}^{-1} .{ }^{19}$ The Zeeman splitting in GaAs and $\mathrm{Au}$ is much smaller than any relevant energy scale in the system and can be neglected, as is also confirmed by numerical simulations.

The SOC due to the structural inversion asymmetry (SIA) at the $\mathrm{Fe} / \mathrm{GaAs}$-interface can be written as $^{20}$

$$
H_{\mathrm{BR}}=\frac{\alpha}{\hbar}\left(\sigma_{x} \pi_{y}-\sigma_{y} \pi_{x}\right) \delta\left(z-z_{l}\right),
$$

where $z_{l}$ denotes the position of the Fe/GaAs interface. As in Refs. 2, 7, and 16 we use the Bychkov-Rashba parameter $\alpha$ as a fitting parameter to reproduce the bias dependence of the TAMR effect; $\alpha=\alpha\left(V_{\mathrm{b}}\right)$. $^{21}$

Finally, the SOC due to the bulk inversion asymmetry (BIA) of the zinc-blende GaAs barrier takes the form ${ }^{22}$

$$
H_{\mathrm{D}}=-\frac{1}{\hbar}\left(\sigma_{x} \pi_{y}+\sigma_{y} \pi_{x}\right) \frac{\partial}{\partial z} \gamma(z) \frac{\partial}{\partial z},
$$

where the bulk Dresselhaus parameter $\gamma=24 \mathrm{eV} \AA^{3}$ in the GaAs barrier and $\gamma=0$ elsewhere. Note that the orbital effects of $B$ are also included in the SOC terms.

With the gauge $\mathbf{A}(z)=[B \sin (\phi) z,-B \cos (\phi) z, 0]$ the Hamiltonian $H$ is translationally invariant in $x$ and $y$ direc- 
tions, and the in-plane wave vector $\mathbf{k}_{\|}=\left(k_{x}, k_{y}, 0\right)$ is a good quantum number. The conductance in the Landauer-Büttiker formalism $^{23}$ is then given as $G=\left[e^{2} S / h(2 \pi)^{2}\right] \int d \mathbf{k}_{\|} T\left(\mathbf{k}_{\|}\right)$, where $S$ is the cross-sectional area of the junction and $T\left(\mathbf{k}_{\|}\right)$ is the total transmission probability (including different spin species) for the transverse wave vector $\mathbf{k}_{\|}$at the Fermi energy $E_{\mathrm{F}}$. We calculate $T\left(\mathbf{k}_{\|}\right)$from the scattering wave functions $;{ }^{24}$ those are obtained numerically from a tightbinding approximation to $H$, using the method of finite differences on a one-dimensional grid with lattice spacing $a=0.01 \mathrm{~nm}$ (Ref. 25) and the recursive Green's-function technique. $^{26}$

In Fig. 2 we compare the results of the numerical simulations on the $B$ dependence of the TAMR with the corresponding experimental data. For this, we fit the parameter $\alpha$ at $B=0.5 \mathrm{~T}$ for every value of the bias voltage $V_{\mathrm{b}}$ to the experimental data. The dependence on $B$ can then be calculated without fitting any further parameter.

Figure 2(a) shows the angular dependence of the TAMR effect for different values of the bias voltage and magnetic field. The numerical simulations show the same trend as the experiment: The magnitude of the TAMR effect decreases with increasing $B$ when the effect is positive, and it increases when the effect is negative. Furthermore, the numerical calculations reproduce the experimentally found change with magnetic field within a factor of 1.5-2. This is an especially satisfying agreement, given the fact that the $B$ dependence is calculated without any fitting parameter. The numerically calculated magnetic field dependence of the TAMR ratio is shown in Fig. 2(b). As the experiment, we find a linear dependence on $B$, with a slope that is nearly independent of $\alpha$, i.e., the bias voltage. Again, the numerics underestimates the slope only by a small factor of 1.5-2.

Having established that our model is able to reproduce both qualitatively and quantitatively the experimental findings, we now develop a phenomenological model to highlight the underlying physics. In Refs. 2, 7, and 16 it was shown that in the absence of a magnetic field, $T\left(\mathbf{k}_{\|}\right)$can be expanded in powers of the SOC in the form $T\left(\mathbf{k}_{\|}\right)$ $=T^{(0)}\left(k_{\|}\right)+T^{(1)}\left(k_{\|}\right) \mathbf{n} \cdot \mathbf{w}\left(\mathbf{k}_{\|}\right)+T^{(2)}\left(k_{\|}\right)\left[\mathbf{n} \cdot \mathbf{w}\left(\mathbf{k}_{\|}\right)\right]^{2}+\cdots, \quad$ where the $T^{(n)}\left(k_{\|}\right)$are expansion coefficients and $\mathbf{w}\left(\mathbf{k}_{\|}\right)$ $=\left[(\tilde{\alpha}-\tilde{\gamma}) k_{y},-(\tilde{\alpha}+\tilde{\gamma}) k_{x}, 0\right]$ the effective spin-orbit field obtained by averaging the spin-orbit field $\mathbf{B}_{\mathrm{SO}}(z), H_{\mathrm{D}}+H_{\mathrm{BR}}$ $=\mathbf{B}_{\mathrm{SO}}(z) \cdot \boldsymbol{\sigma}$, over the unperturbed states of the system. The effective spin-orbit parameters are given by $\tilde{\alpha}=\alpha f_{\alpha}\left(k_{\|}\right)$and $\tilde{\gamma}=\gamma f_{\gamma}\left(k_{\|}\right)$. To second order in the SOC, the conductance was then found as

$$
G(\phi)=G_{0}+g^{(2)} \alpha \gamma \cos (2 \phi),
$$

where $G_{0}$ is the angular-independent part of the conductance and $g^{(2)}$ a coefficient that is independent of the spin-orbit parameters (for details see Refs. 2 and 16).

In the presence of a magnetic field, the transmission can still be expanded in powers of the SOC, albeit with $B$-dependent coefficients $T_{B}^{(n)}\left(\mathbf{k}_{\|}\right)$and spin-orbit field $\mathbf{w}_{B}\left(\mathbf{k}_{\|}\right)$. Below, we derive approximate relations for $T_{B}^{(n)}\left(\mathbf{k}_{\|}\right)$and $\mathbf{w}_{B}\left(\mathbf{k}_{\|}\right)$, valid to linear order in $B$, in terms of their counterparts at $B=0, T^{(n)}\left(k_{\|}\right)$and $\mathbf{w}\left(\mathbf{k}_{\|}\right)$.

First, we consider the orbital effects of $B$ on the kinetic-
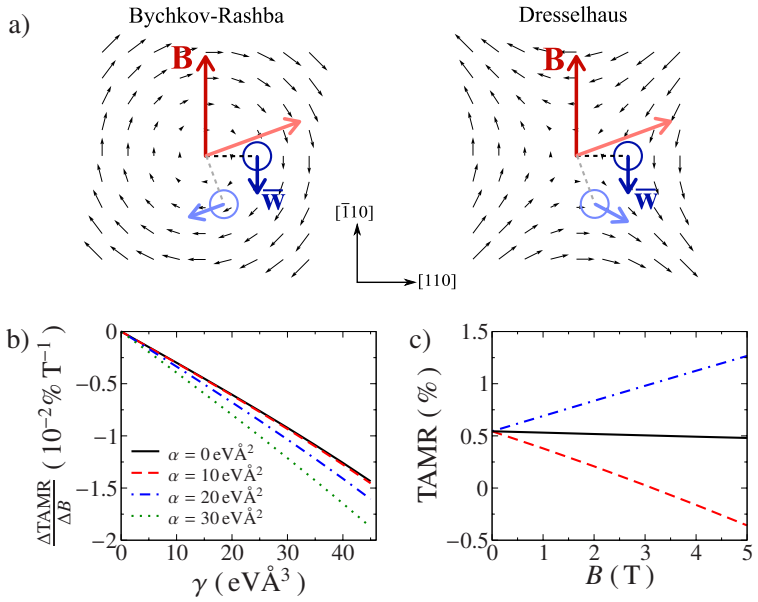

FIG. 3. (Color online) (a) Schematic picture of the influence of $\mathbf{B}$ on the TAMR: $\mathbf{B}$ and $\overline{\mathbf{w}}=\int d \mathbf{k}_{\|} T_{B}^{(1)} \mathbf{w}_{B}$ are shown in relation to the effective Bychkov-Rashba and Dresselhaus spin-orbit fields. The shift of the transmission maximum is indicated by a blue circle; the situation for two different angles $\phi$ is shown in dark and light color. (b) Slope of the $B$ dependence of the TAMR effect, $\Delta$ TAMR $/ \Delta B$, as a function of the Dresselhaus parameter $\gamma$ for various values of $\alpha$. (c) $B$ dependence of the TAMR effect when $\mathbf{A}$ is only included in the kinetic term (red dashed line), only in the spin-orbit term (blue dash-dotted line), or in both terms (black solid line).

energy term of the Hamiltonian. The kinetic energy associated with $\mathbf{k}_{\|}$increases the effective barrier height, and hence $T\left(\mathbf{k}_{\|}\right)$is sharply peaked at $\mathbf{k}_{\|}=0$ in the absence of a magnetic field. For $B \neq 0$ however, the effective barrier height is smallest for an in-plane wave vector $\mathbf{k}_{\|, 0}$ with $\left\langle\left[k_{x, 0}+e B / \hbar \sin (\phi) z\right]^{2}\right\rangle=0$ and $\left\langle\left[k_{y, 0}-e B / \hbar \cos (\phi) z\right]^{2}\right\rangle=0$, where $\langle\ldots\rangle$ denotes a quantum mechanical average. Thus, the maximum of the transmission is shifted to $\mathbf{k}_{\|, 0}=\left[-b_{1} B \sin (\phi), b_{1} B \cos (\phi), 0\right]$, where $b_{1}$ depends on $\langle z\rangle$ and $\left\langle z^{2}\right\rangle$, and hence we assume $T_{B}^{(n)}\left(\mathbf{k}_{\|}\right)$ $\approx T^{(n)}\left(\sqrt{\left(k_{x}-k_{x, 0}\right)^{2}+\left(k_{y}-k_{y, 0}\right)^{2}}\right)$. This shift can be interpreted as an effect of the Lorentz force. In addition to the shift of the maximum, the overall transmission decreases. ${ }^{15}$ However, this decrease is quadratic in $B$ and will consequently be neglected. Apart from $T_{B}\left(\mathbf{k}_{\|}\right)$, also the effective spin-orbit field is shifted in momentum space, $\mathbf{w}_{B}\left(\mathbf{k}_{\|}\right)$ $\approx \mathbf{w}\left(k_{x}+b_{2} B \sin (\phi), k_{y}-b_{2} B \cos (\phi)\right)$, where $b_{2}$ is a constant that depends on $\langle z\rangle$ only, as the SOC terms are linear in momentum. Therefore we can in general expect $b_{1} \neq b_{2}$.

With these approximations we can now obtain the magnetic field corrections to the conductance of Eq. (5) by evaluating $\int d \mathbf{k}_{\|} T\left(\mathbf{k}_{\|}\right)$in orders of the SOC. The zeroth-order term remains unchanged upon integration, and the corrections to the second-order term are quadratic in $B$, thus being neglected. In contrast, the first-order term that vanishes in the absence of a magnetic field ${ }^{2,7,16}$ gives a contribution linear in $B$ :

$$
\frac{e^{2} S}{h(2 \pi)^{2}} \mathbf{n} \cdot \int d \mathbf{k}_{\|} T_{B}^{(1)}\left(\mathbf{k}_{\|}\right) \mathbf{w}_{B}\left(\mathbf{k}_{\|}\right)=g_{\alpha}^{(1)} \alpha B-g_{\gamma}^{(1)} \gamma B \cos (2 \phi),
$$

where we used the approximations of the previous paragraph and the fact that terms linear in $\mathbf{k}_{\|}$vanish 
upon integration. ${ }^{2,7,16}$ The coefficients $g_{\alpha, \gamma}^{(1)}$ $=\left(e^{2} S / h(2 \pi)^{2}\right)\left(b_{1}-b_{2}\right) \int d \mathbf{k}_{\|} T^{(1)}\left(k_{\|}\right) f_{\alpha, \gamma}\left(k_{\|}\right)$do not depend on the spin-orbit parameters. We find a different angular dependence for the Bychkov-Rashba and Dresselhaus SOC due to different symmetries of the spin-orbit fields, as shown in Fig. 3(a): the Bychkov-Rashba field exhibits rotational symmetry leading to an angular-independent contribution, whereas the interplay of $B$ and the Dresselhaus field leads to an angular dependence with uniaxial symmetry.

The total conductance in a magnetic field is then

$$
G(\phi, B)=G_{0}+g_{\alpha}^{(1)} \alpha B+\left(g^{(2)} \alpha \gamma-g_{\gamma}^{(1)} \gamma B\right) \cos (2 \phi)
$$

valid up to second order in the SOC. The magnetic field dependence of the TAMR ratio is then

$$
\text { TAMR } \propto g^{(2)} \alpha \gamma-g_{\gamma}^{(1)} \gamma B,
$$

where we can deduce from the numerical results that the coefficients $g^{(2)}, g_{\gamma}^{(1)}>0$. Equation (8) reproduces all the characteristic features of the TAMR observed in experiment: a linear $B$ dependence with a bias $(\alpha)$-independent slope. Note that the interplay of Dresselhaus SOC in the barrier and the orbital effect of the magnetic field leads to an independent contribution to the TAMR effect which turns out to have the same uniaxial symmetry as the TAMR effect in the absence of $B$.
Finally, we verify some aspects of the phenomenological model by comparing to numerical simulations. In Fig. 3(b) we show the slope $\triangle T A M R / \triangle B$ as a function of the Dresselhaus parameter $\gamma$ that is predicted to be linear in $\gamma$ and independent of $\alpha$ [Eq. (8)]. Indeed, we find a nearly linear dependence on $\gamma$ and only a weak dependence on $\alpha$, presumably originating from higher orders in the SOC expansion. Furthermore, the coefficient $g_{\gamma}^{(1)}$ in Eq. (8) depends on $\left(b_{1}-b_{2}\right)$, i.e., opposing contributions from the kinetic and the SOC term. Figure 3(c) shows the results of simulations where the magnetic vector potential is included only in the kinetic term (dashed line), only in the SOC term (dasheddotted line), and in both (solid line). When the magnetic field is included in one term only, we find large TAMR effects with opposite sign that nearly cancel in the full Hamiltonian, yielding the small signal observed in experiment and in the numerics.

In summary, our experiments and theoretical considerations indicate that the interplay of the orbital effects of a magnetic field and the Dresselhaus SOC in a tunnel barrier leads to a contribution to the TAMR effect with uniaxial symmetry. This effect is predicted to an intrinsic feature of semiconductor barriers with BIA and not limited to the studied $\mathrm{Fe} / \mathrm{GaAs} / \mathrm{Au}$ tunnel junction.

We gratefully acknowledge financial support by the German Science Foundation via SFB 689.
${ }^{1}$ I. Žutić, J. Fabian, and S. Das Sarma, Rev. Mod. Phys. 76, 323 (2004).

${ }^{2}$ J. Fabian et al., Acta Phys. Slov. 57, 565 (2007).

${ }^{3}$ C. Chappert, A. Fert, and F. N. Van Dau, Nature Mater. 6, 813 (2007).

${ }^{4}$ C. Gould, C. Ruster, T. Jungwirth, E. Girgis, G. M. Schott, R. Giraud, K. Brunner, G. Schmidt, and L. W. Molenkamp, Phys. Rev. Lett. 93, 117203 (2004).

${ }^{5}$ C. Rüster, C. Gould, T. Jungwirth, J. Sinova, G. M. Schott, R. Giraud, K. Brunner, G. Schmidt, and L. W. Molenkamp, Phys. Rev. Lett. 94, 027203 (2005).

${ }^{6}$ H. Saito, S. Yuasa, and K. Ando, Phys. Rev. Lett. 95, 086604 (2005).

${ }^{7}$ J. Moser, A. Matos-Abiague, D. Schuh, W. Wegscheider, J. Fabian, and D. Weiss, Phys. Rev. Lett. 99, 056601 (2007).

${ }^{8}$ B. G. Park, J. Wunderlich, D. A. Williams, S. J. Joo, K. Y. Jung, K. H. Shin, K. Olejnik, A. B. Shick, and T. Jungwirth, Phys. Rev. Lett. 100, 087204 (2008).

${ }^{9}$ M. Ciorga, M. Schlapps, A. Einwanger, S. Geißler, J. Sadowskil, W. Wegscheider, and D. Weiss, New J. Phys. 9, 351 (2007).

${ }^{10}$ A. D. Giddings, M. N. Khalid, T. Jungwirth, J. Wunderlich, S. Yasin, R. P. Campion, K. W. Edmonds, J. Sinova, K. Ito, K.-Y. Wang, D. Williams, B. L. Gallagher, and C. T. Foxon, Phys. Rev. Lett. 94, 127202 (2005).

${ }^{11}$ K. I. Bolotin, F. Kuemmeth, and D. C. Ralph, Phys. Rev. Lett. 97, 127202 (2006).

${ }^{12}$ A. N. Chantis, K. D. Belashchenko, E. Y. Tsymbal, and M. van Schilfgaarde, Phys. Rev. Lett. 98, 046601 (2007).

${ }^{13}$ J. D. Burton, R. F. Sabirianov, J. P. Velev, O. N. Mryasov, and E. Y. Tsymbal, Phys. Rev. B 76, 144430 (2007).
${ }^{14}$ M. N. Khan, J. Henk, and P. Bruno, J. Phys.: Condens. Matter 20, 155208 (2008).

${ }^{15}$ L. Eaves, K. W. H. Stevens, and F. W. Sheard, in The Physics and Fabrication of Microstructures and Microdevices, edited by M. Kelly and C. Weisbuch (Springer, Berlin, 1986).

${ }^{16}$ A. Matos-Abiague and J. Fabian, Phys. Rev. B 79, 155303 (2009).

${ }^{17}$ E. C. Stoner, Proc. R. Soc. London, Ser. A 169, 339 (1939).

${ }^{18}$ Magnetic Properties of Metals: d-Element, Alloys, and Compounds, edited by H. P. J. Wijn (Springer, Heidelberg, 1991).

${ }^{19}$ N. W. Ashcroft and N. D. Mermin, Solid State Physics (Saunders College, Philadelphia, 1988).

${ }^{20}$ Y. A. Bychkov and E. I. Rashba, J. Phys. C 17, 6039 (1984); JETP Lett. 39, 78 (1984).

${ }^{21}$ This interpretation is also supported by density functional theory: M. Gmitra, A. Matos-Abiague, C. Ambrosch-Draxl, and J. Fabian, arXiv:0907.4149 (unpublished).

${ }^{22}$ G. Dresselhaus, Phys. Rev. 100, 580 (1955).

${ }^{23}$ S. Datta, Electronic Transport in Mesoscopic Systems (Cambridge University Press, Cambridge, 2002).

${ }^{24}$ The gauge $\mathbf{A}(z)$ does not allow for plane-wave solutions in the contacts. Hence, we switch off $B$ away from the barrier. Since the cyclotron radius in $\mathrm{Fe}$ and $\mathrm{Au}$ is much larger than any relevant length scale $\left(l_{\text {cycl }} \approx 1.5 \mu \mathrm{m}\right.$ at $\left.5 \mathrm{~T}\right)$, the exact position of this switching is not important.

$25 a$ is chosen very small to accurately approximate the $\delta$ function of Eq. (3) by a rectangular function with unit area and width $2 a$, centered at the $\mathrm{Fe} / \mathrm{GaAs}$-interface.

${ }^{26}$ M. Wimmer, M. Scheid, and K. Richter, arXiv:0803.3705, Encyclopedia of Complexity and System Science (Springer, 2009). 